(1)

George Fox

UNIVERSITY
Digital Commons @ George Fox University

Faculty Publications - Grad School of Clinical

Psychology

Graduate School of Clinical Psychology

2016

Physician-Patient Communication About Sexual Functioning in

Patients with Multiple Sclerosis

Lara M. Stepleman

Eliot J. Lopez

Lauren A. Stutts

William $\mathrm{H}$. Hudson

Marie-Christine Goodworth

See next page for additional authors

Follow this and additional works at: https://digitalcommons.georgefox.edu/gscp_fac

Part of the Psychology Commons 


\section{Authors}

Lara M. Stepleman, Eliot J. Lopez, Lauren A. Stutts, William H. Hudson, Marie-Christine Goodworth, and Rebecca Han 


\title{
Physician-Patient Communication About Sexual Functioning in Patients with Multiple Sclerosis
}

\author{
Lara M. Stepleman ${ }^{1}$ Eliot J. Lopez ${ }^{1} \cdot$ Lauren A. Stutts $^{2}$ • \\ William H. Hudson ${ }^{3}$ - Marie-Christine Rutter Goodworth ${ }^{4}$. \\ Rebecca Rahn 5
}

\begin{abstract}
Sexual dysfunction is quite common among individuals with multiple sclerosis (MS); however, severity of dysfunction alone does not account for the tremendous variation in sexual satisfaction across individuals living with MS. Individual characteristics, relationships with intimate partners, and environmental factors all likely contribute to the multidimensional experience of sexual satisfaction. Health care provider variables, including how one communicates with providers about sexual concerns, may also be influential. The purpose of this study was to examine factors that are associated with patients' sex-related communications with their MS physicians and to overall patient sexual satisfaction. Individuals in an MS clinic $(n=73)$ completed a survey packet which included measures of physical and mental health, sexual dysfunction, sexual satisfaction, sex communication, health care provider relationships, and health care satisfaction. Findings suggest that while more than half of patients with MS reported experiencing sexual dysfunction, only a third of patients indicated addressing their sexual concerns with their physician during the past year. Interestingly, the frequency of communication about sexual concerns was associated with satisfaction with physician variables, whereas selfefficacy for these interactions was associated with emotional health variables. These results indicate that when considering interventions to increase confidence for communication and frequency of communication that differing factors may be taken into account.
\end{abstract}

Lara M. Stepleman

1steplem@gru.edu

1 Department of Psychiatry and Health Behavior, Medical College of Georgia, Augusta University, 997 St. Sebastian Way, Augusta, GA 30912, USA

2 Department of Psychology, Davidson College, Davidson, NC, USA

3 Department of Psychiatry and Behavioral Sciences, Emory University, Atlanta, GA, USA

4 Graduate Department of Clinical Psychology, George Fox University, Newberg, OR, USA

5 Augusta Multiple Sclerosis Center, Augusta University, Augusta, GA, USA 
Keywords Multiple sclerosis - Sexual functioning - Physician-patient communication · Sexual satisfaction · United States

\section{Introduction}

Multiple sclerosis (MS) is one of the most common neurologic disorders in adults, affecting approximately 250,000-350,000 people in the United States [1]. Widely thought to be an autoimmune disease, MS is characterized pathologically by the development of focal areas of demyelination in the white matter of the brain and spinal cord and in the optic nerve [2]. Physical symptoms can include fatigue, numbness, gait disorder, balance/coordination problems, bladder/bowel dysfunction, vision problems, vertigo, pain, spasticity, and sexual dysfunction. In addition, approximately $45-65 \%$ of patients with MS develop cognitive disabilities that affect higher-level brain functions such as learning, memory, organization, and problem-solving [3]. Individuals with MS may also experience emotional changes including depression, mood swings, and irritability [4].

The course of the disease varies considerably and is rather unpredictable; however, about $85 \%$ of individuals with MS experience a relapse-remitting course, which does not progress between attacks, at least initially. Further, other individuals experience a progressive MS course, which is defined as progression of disability from onset [2]. After an initial episode, patients may experience an interval of months to years before further symptoms arise. Although at least some degree of recovery from an acute attack can be expected, approximately $70 \%$ of those living with MS report walking as the most challenging aspect of the disease [5]. Since there is no cure for MS, treatment consists of attempting to slow progression via disease modifying agents and managing symptoms. Although many of these symptoms are physiological, a wide range of psychosocial problems can significantly disrupt the lives of people living with MS such as affecting their emotions, relationships, and quality of life. In particular, sexual dysfunction presents both physically and psychosocially $[6,7]$.

\section{MS and Sexual Functioning}

The incidence of sexual dysfunction is high in the MS population relative to the general population and to other individuals living with a chronic illness [8]. A number of studies have described the prevalence rates of sexual dysfunction in MS, with the incidence ranging from 50 to $90 \%$ in men and from 40 to $80 \%$ in women [9-14]. Given the importance of sexuality and intimacy in relationships, sexual dysfunction can significantly disrupt quality of life for individual living with MS [15, 16]. Relative to other health issues in MS, research on sexuality and sexual functioning has received less attention, especially in regards to psychosocial factors such as impact of sexual dysfunction on relationship interaction and/or self-image [17].

Foley et al. [18] described a model dividing MS-related sexual dysfunction into primary, secondary, and tertiary sexual dysfunction. Primary sexual dysfunction occurs due to MS-related neurologic changes that directly affect sexual feelings/response. Men may experience impaired genital sensation, decreased libido, inability to maintain an erection, and decreased frequency and/or force of ejaculation, while women may experience decreased libido, altered genital sensation, decreased vaginal lubrication, and decreased frequency and/or intensity of orgasm. Secondary sexual dysfunction refers to MS-related physical changes that affect the sexual response indirectly, such as fatigue, spasticity, 
bladder/bowel dysfunction, coordination and mobility problems, cognitive difficulties, and pain/discomfort in non-genital areas. Finally, tertiary sexual dysfunction refers to the psychological, emotional, social, and cultural aspects of MS that affect sexuality. Tertiary symptoms can include negative changes in self-image, mood disturbances including depression and anger, feeling less confident about one's sexuality, and difficulty communicating with one's partner among others [18, 19]. Given the frequency of sexual difficulties in MS and its multifactorial presentation, effective patient-provider communication about sex and intimacy issues may serve to improve management of sexual dysfunction $[20,21]$.

\section{Patient Care Variables and Ability to Communicate}

A number of studies examining the effects of patient care variables on sexual communication have illustrated the inherent complexity of the patient-health care provider interaction. This complexity is especially true with regard to establishing a dialogue about sexual satisfaction [22-25] Sexual health concerns may not be addressed sufficiently if there are difficulties related to any combination of the following: the ability of the health care provider to communicate, the patient's comfort with the provider, or the patient's confidence in talking with the provider. Moreover, these factors may interact in ways that confound the problem, further inhibiting potentially valuable discussions concerning the patient's sexual satisfaction and thus quality of life [26].

A health care provider's ability to communicate effectively about sexual health concerns with patients living with a chronic illness has been examined in the literature though few research studies have instigated patient-provider communication specific to MS. Notably, in one qualitative study, Rubin [21] identified that MS providers believed that conversations about sexual functioning might be viewed as intrusive. They also indicated that lack of provider information and training served as barriers to communication. In one study, surveys from two different rehabilitation centers working with physical problems (e.g. spinal cord injury, stroke, MS) revealed that $67 \%$ of rehabilitation professionals considered sexuality an important topic to discuss, though only $12 \%$ considered themselves sufficiently trained to do so [27].

In addition to a provider's ability to communicate, a patient's level of comfort with the provider has been shown to influence patient-provider communication about sexual concerns. Perceived positive relationships with physicians have been found to be essential to voicing sexual concerns, and patients who did not feel respected by their physicians were unlikely to address them [28]. In fact, Lew et al. [13] found that in a sample of women with MS, only $2.2 \%$ reported ever discussing their sexual concerns with a physician. Patient reluctance to bring up sexual health concerns also may lead health care providers to erroneously believe that a patient has either adapted to changes in sexual functioning or that he or she does not have any sexual concerns [29]. Likewise, having implicit trust in one's physician may lead patients to believe that if a physician does not raise an issue, then it is not a valid concern [28, 30]. By inquiring about sexual concerns, health care professionals give patients "permission" to address these issues in a confidential and nonjudgmental setting [29]. In addition to the many useful findings from the robust cancer literature on the topic, further exploration of patient-provider communication in other chronic illnesses, including MS, is still needed. 


\section{Mental Health Variables and Ability to Communicate}

Although little attention has been given to the role of mood disorders in sexual communication, investigators have examined the relationship between mood disorders and more general health care communication in other medical populations. For example, patients with depressive symptoms also report more unmet expectations and less satisfaction with their care than patients without depression [31,32]. Furthermore, physicians are more likely to describe their visits with anxious or depressed patients as "difficult," which could also impact the amount of patience a physician might have for more sensitive or challenging conversations [33].

Emotional difficulties experienced by people with MS have been well documented $[34,35]$. Depression is the most common mood disorder in patients with MS, more common than in other neurological disorders [36], with a lifetime prevalence co-morbidity of 42-54\% [37-39]. Given evidence that depression may alter communication quality and satisfaction in medical settings, it is essential that the influence of depression be further examined with regard to partner and physician sex-related communication in MS.

\section{Purpose of Study}

Despite the large number of people living with multiple sclerosis and the serious physical and emotional symptoms that impact sexuality and sexual functioning, little is known about variables contributing to a patient's perceived ability to communicate with his or her physician about sex and potential subsequent impact on sexual satisfaction. The purpose of our study was to examine sexual health, health care communication, and MS-related variables within the context of sexual health care communication and overall sexual satisfaction in persons with MS. Better communication would ideally help the patient initiate earlier intervention for his/her MS related sex and intimacy problems. By further understanding influences on a patient's ability to communicate about MS and its impact on sexuality and sexual satisfaction, programs may be developed to facilitate effective communication among patients and health care providers.

\section{Methods}

\section{Participants}

Participants included 73 patients recruited from a regional Multiple Sclerosis Center located in the southeastern United States. The mean age of the patients was 43.53 $(S D=10.24)$, ranging from 20 to 65 years of age. The majority of the participants were female $(87.7 \%)$, Caucasian $(60.3 \%)$, married (66.7\%), college educated $(56.1 \%)$, and currently unemployed/disabled (55.6\%). Demographic information about the participants is detailed in Table 1. Approximately $73.6 \%$ of the participants had relapse-remitting MS, $6.9 \%$ had secondary progressive MS, $1.4 \%$ had primary progressive MS, $2.8 \%$ had progressive relapsing, and, for $15.3 \%$, the type of MS was unspecified. Patients on average were 8 years from diagnosis. 
Table 1 Sample demographic characteristics

\begin{tabular}{lrc}
\hline & $N$ & $\%$ of total \\
\hline Sex & & \\
Female & 64 & 87.70 \\
Male & 9 & 12.30 \\
Race & & \\
Caucasian & 44 & 60.30 \\
African American & 27 & 37.00 \\
Latino(a)/Hispanic & 1 & 1.40 \\
Other & 1 & 1.40 \\
Relationship/partner status & & \\
Single/not interested in dating & 5 & 6.90 \\
Single/interested in dating & 7 & 9.30 \\
Casually dating & 2 & 2.80 \\
Committed relationship/not living together & 7 & 9.70 \\
Committed relationship/living together & 3 & 4.20 \\
Married & 48 & 66.70 \\
Education & & \\
No high school & 3 & 4.10 \\
Some high school & 1 & 1.40 \\
GED/high school & 12 & 16.40 \\
Some college & 16 & 21.90 \\
2-year degree & 12 & 16.40 \\
4-year degree & 17 & 23.30 \\
Master's degree & 10 & 13.70 \\
Prof degree & 2 & 2.70 \\
Employment & & \\
Unemployed/disabled & 40 & 55.60 \\
Retired & 4 & 5.60 \\
Part-time employment & 4 & 5.60 \\
Full-time employment & 33.30 \\
\hline & & \\
\hline & & \\
& & \\
& & \\
& & \\
& & \\
& &
\end{tabular}

\section{Procedure}

The Institutional Review Board of the university with which the MS Center is affiliated approved the following study procedures. During the recruitment period, all eligible adult patients who presented for regularly scheduled appointments at the MS Center were invited to participate in the study. Eligibility requirements included a documented definitive MS diagnosis as well as confirmation by the patients' neurologist that the individual does not have significant cognitive impairment. Patients were informed of the voluntary nature of the study and of the study goal to examine sexuality and intimacy in people living with MS. Verbal and written informed consent were obtained from participants prior to participation. The survey packet generally took 25-35 min to complete during the medical visit. Patients were orally administered the survey by an investigator, at patient request, when physical limitations prohibited independent completion. Each participant received a five dollar gift card after their study participation ended. 


\section{Measures}

\section{Demographic Questionnaire}

Nine questions were included in the survey packet to gather demographic data related to race, gender, age, education, relationship status, and duration of MS diagnosis.

\section{Patient Determined Disease Steps (PDDS)}

This single-item self-assessment of MS-related disability asks participants to indicate their primary level of mobility from the following options: normal, mild disability, moderate disability, gait disturbance, early cane, late cane, bilateral support, or confined to a wheelchair [40]. Descriptions of each category are provided to better assist individuals in making this assessment. The PDDS is strongly correlated with the gold-standard, physician-administered Expanded Disability Status Scale (EDSS) [41].

\section{Mental Health Inventory (MHI)}

This 18-item instrument provides assessment across several domains of mental health, including anxiety, depression, behavioral control (e.g., emotional stability), positive affect, and general distress [42]. The MHI is one of the components of the Multiple Sclerosis Quality of Life Inventory [43]. Items are scored on a six-point likert-type scale ranging from 1 (None of the time) to 6 (All of the time). The total score, as well as subscale scores, were weighted to range from 0 to 100 . Higher scores indicate better mental health. The items comprising the MHI total score were found to have a coefficient alpha of .82 and good convergent and discriminant validity [42].

\section{Multiple Sclerosis Intimacy and Sexuality Questionnaire-19 (MSISQ-19)}

This measure is a 19-item assessment of the perceived influence of MS symptoms on sexual activity, sexual satisfaction, and the overall quality of intimate relationships [44]. There are three subscales corresponding to primary, secondary, and tertiary sexual dysfunction. Participants rate sexual concerns on a scale from 1 (never) to 5 (always). Higher scores indicate greater levels of sexual dysfunction. The MSISQ-19 has been shown to have a high internal consistency for the total score $(\alpha=.91)$ and for the primary $(\alpha=.82)$, secondary $(\alpha=.85)$ and tertiary $(\alpha=.87)$ subscales (Sanders et al., 2000). The MSISQ19 also has concurrent validity with multiple other measures (e.g., marital satisfaction, psychological distress, and global sexual dysfunction) [44].

\section{Sexual Satisfaction Scale (SSS)}

The SSS is a 4-item instrument of sexual satisfaction and one component of the Multiple Sclerosis Quality of Life Inventory [43, 45]. First, participants answer yes or no to whether they have one primary partner (survey is to be skipped if they do not). Subsequently, they rate their satisfaction in the past 4 weeks on a scale from 1 (Extremely Satisfied) to 6 (Extremely Dissatisfied) for the following items: the amount of affection expressed physically, variety of sexual activities engaged in, sexual relationship in general, and perceived partner satisfaction regarding the sexual relationship. Total scale scores can 
range from 4 to 24 with higher scores indicating greater dissatisfaction. This measure has been shown to have a Cronbach's alpha of .91 and has good face validity [45].

\section{Patient Satisfaction Questionnaire (PSQ-18)}

This 18-item measure is the short form of the PSQ-III and examines seven dimensions of satisfaction with medical care: general satisfaction, technical quality, interpersonal manner, communication, financial aspects, time spent with doctor, and accessibility and convenience, which can each be used as independent subscales [46]. Participants rate each item on a 5 -point ratings scale $(1=$ strongly agree to $5=$ strongly disagree $)$. Items are reverse scored and totaled for each subscale; higher numbers indicate higher satisfaction. The PSQ-18 has demonstrated good internal consistency and is strongly correlated with the original long form PSQ-III. [46].

\section{Perceived Self-Efficacy in Patient-Physician Interactions-Sex (PEPPI-S)}

This measure is an author-modified version of the 5-item PEPPI, a measure of patients' self-efficacy for acquiring physician information and attention regarding medical concerns [47]. The authors did minor item revisions, making the instrument specific to sexual health concerns. Participants rated their confidence on a scale from 0 (not confident) to 10 (most confident) in their ability to: (1) know what questions to ask the doctor about sexual health concerns, (2) get the doctor to answer all their questions about sexual health concerns, (3) make the most of their visits with the doctor about sexual health concerns, (4) get the doctor to take their sexual health concerns seriously, and (5) get the doctor to do something about their sexual health concerns. The scores are totaled and range from 0 to 50 with higher numbers indicating greater perceived efficacy. The PEPPI has demonstrated excellent reliability ( $\alpha=0.93$ ) as well as discriminant and convergent validity [47]. Our modified version of the instrument, the PEPPI-S, had strong internal consistency with was a coefficient alpha of .97 .

\section{Frequency of Sex-Related Communication and Behaviors}

This 9-item author-derived instrument was designed to assess the frequency of patient communication about sexual concerns with their physician and primary partner over the last 7 days, 30 days, and 1 year and the frequency of sexual activity with their primary partner for the same time intervals; analyses conducted in this study focused solely on communication within the last year. Items were scored on a six-point likert-type scale, ranging from 1 (None) to 6 (More than 20 times). Scores for each subscale were averaged to produce an approximation of how often a patient engaged in these specific behaviors. Higher scores were reflective of greater engagement in these activities.

\section{Results}

All data analyses were performed using SPSS for Windows (Version 23). Descriptive statistics, including coefficient alphas, for our measures are detailed in Table 2. Descriptive statistics were conducted to summarize the demographic and background characteristics of the sample. For several of the demographic variables, categories were collapsed due to low 
Table 2 Measure descriptive statistics

\begin{tabular}{|c|c|c|c|c|}
\hline Measure & Possible range & $\begin{array}{l}\text { Sample } \\
\text { average }(S D)\end{array}$ & $\begin{array}{l}\text { Sample } \\
\text { Range }\end{array}$ & $\begin{array}{l}\text { Coefficient } \\
\text { Alpha }\end{array}$ \\
\hline MHI & $\begin{array}{l}0-100 \text { (higher scores }=\text { better } \\
\text { mental health) }\end{array}$ & & & \\
\hline Anxiety & & $61.21(22.38)$ & $12-100$ & 0.84 \\
\hline Depression & & $65.41(23.30)$ & $0-100$ & 0.9 \\
\hline Behavioral control & & $73.37(21.51)$ & $10-100$ & 0.5 \\
\hline Positive affect & & $60.21(19.53)$ & $0-100$ & 0.78 \\
\hline Total & & $65.08(18.95)$ & $12.22-96.67$ & 0.93 \\
\hline MSISQ & $\begin{array}{l}1-5 \text { (higher scores }=\text { more sexual } \\
\text { dysfunction) }\end{array}$ & & & \\
\hline Primary & & $2.99(1.08)$ & $1-5$ & 0.88 \\
\hline Secondary & & $2.34(0.81)$ & $1-4$ & 0.84 \\
\hline Tertiary & & $2.27(0.98)$ & $1-4$ & 0.81 \\
\hline Total & $19-95$ & $49.07(14.42)$ & $21-83$ & 0.9 \\
\hline SSS & $\begin{array}{l}\text { 4-24 } \text { (higher scores }=\text { more } \\
\text { dissatisfaction) }\end{array}$ & $11.23(6.54)$ & $4-24$ & 0.88 \\
\hline PSQ & $\begin{array}{l}1-5 \text { (higher scores }=\text { higher } \\
\text { satisfaction) }\end{array}$ & & & \\
\hline General satisfaction & & $4.03(0.90)$ & $1-5$ & 0.76 \\
\hline Technical quality & & $4.11(0.77)$ & $1.25-5$ & 0.8 \\
\hline Interpersonal & & $4.40(0.67)$ & $1.5-5$ & 0.64 \\
\hline Communication & & $4.05(0.83)$ & $1-5$ & 0.58 \\
\hline Financial aspects & & $3.36(1.12)$ & $1-5$ & 0.6 \\
\hline Time & & $3.96(0.94)$ & $1-5$ & 0.79 \\
\hline Access/convenience & & $3.53(0.87)$ & $1-5$ & 0.71 \\
\hline Total average & & $3.92(0.65)$ & $1.61-5$ & 0.9 \\
\hline PEPPI-S total & $\begin{array}{l}0-50 \text { (higher scores }=\text { higher } \\
\text { perceived efficacy) }\end{array}$ & $34.79(13.14)$ & $0-50$ & 0.97 \\
\hline
\end{tabular}

frequency within some of the groups. Specifically, ethnicity was divided into Caucasian and ethnic minority for analysis; relationship status was divided into "spouse/partner in residence" and "no spouse/partner in residence;" education was recoded into three categories: "high school and lower," "some college but $<$ a 4 year degree," and a "4 year degree or higher;" and employment was collapsed into "employed" and "not employed."

\section{Indicators of MS Severity and Sexual Functioning}

The largest proportion of participants described themselves as having some degree of gait disturbance, as measured by the PDDS (23.3\%; see Table 3). Patient MS course (relapseremitting or progressive) was related to PDDS score, $t(59)=2.69, p<.01$. Patients with a progressive MS on average had higher self-ratings of disability $(M=4.25, S D=2.05)$ than did those with a relapse-remitting MS $(M=2.40, S D=1.78)$. Similarly, patients 
Table 3 Self-described degree of mobility

\begin{tabular}{lcr}
\hline Descriptor & Frequency & $\%$ \\
\hline Normal & 11 & 15.1 \\
Mild disability & 8 & 11.0 \\
Moderate disability & 12 & 16.4 \\
Gait disturbance & 17 & 23.3 \\
Early cane & 7 & 9.6 \\
Late cane & 9 & 12.3 \\
Bilateral support & 4 & 5.5 \\
Confined to a wheelchair & 5 & 6.8 \\
\hline
\end{tabular}

who had been diagnosed longer with MS reported higher scores on the PDDS $(r=.39$, $p<.01)$.

Regarding sexual dysfunction, patients with progressive MS reported more sexual dysfunction overall $[t(59)=2.37, p<.05]$ than those patients with relapsing-remitting MS. Further, patients with progressive MS also reported greater secondary symptoms of sexual dysfunction $[t(59)=2.12, p<.02]$ than their relapsing-remitting counterparts. Similar trends $(p<.10)$ were found in the MSISQ-19 primary and tertiary subscales.

Regarding frequency of engaging in sexual behavior, $70 \%$ of participants reported engaging in sexual activity at least one to two times in the last 7 days, $84.7 \%$ engaged in sexual activity at least one to two times in the last 30 days, and $96.6 \%$ engaged in sexual activity at least one to two times in the last year (see Table 4). Frequency of sexual behavior was not associated with MS severity as measured by the PDDS, time since diagnosis, MS subtype, and severity of MS sexual dysfunction as measured by the MSISQ19 and its subscales.

\section{Communication Frequency and Self-Efficacy Regarding Sexual Concerns}

Estimated frequency of and perceived confidence for sex-related communications with the physician were assessed. Regarding frequency, $32.4 \%$ of patients indicated that they talked to their physician about sexual concerns in the last year (see Table 5). Of the patient demographics, only patient age and the duration of patients' current intimate relationships were associated with the frequency of communicating about sexual concerns with their physician; younger age $(r=-.27, p<.05)$ and shorter relationship duration $(r=-.29$,

Table 4 Frequency of sexual activity with partner

\begin{tabular}{lllc}
\hline & Last 7 days $(\%)$ & Last 30 days $(\%)$ & Last 12 months $(\%)$ \\
\hline 0 times & 30.0 & 15.3 & 3.3 \\
$1-2$ times & 40.0 & 16.9 & 6.7 \\
$3-5$ times & 25.0 & 22.0 & 10.0 \\
$6-10$ times & 3.3 & 30.5 & 13.3 \\
$10-20$ times & 1.7 & 11.9 & 13.3 \\
More than 20 times & 0 & 3.4 & 53.3 \\
\hline
\end{tabular}


Table 5 Frequency of patient-physician communication

\begin{tabular}{lccc}
\hline & Last 7 days $(\%)$ & Last 30 days $(\%)$ & Last 12 months $(\%)$ \\
\hline 0 times & 88.7 & 85.9 & 67.6 \\
$1-2$ times & 7.0 & 12.7 & 16.9 \\
$3-5$ times & 1.4 & 0 & 9.9 \\
$6-10$ times & 0 & 0 & 2.8 \\
$10-20$ times & 2.8 & 1.4 & 2.8 \\
More than 20 times & 0 & 0 & 0 \\
\hline
\end{tabular}

$p<.05)$ were associated with more frequent communication in the last year. Several subscales from the PSQ were also associated with greater sexual communication with a physician in the past year, including general satisfaction with care $(r=.37, p<.01)$, time spent with doctor, $(r=.32, p<.01)$, and communication $(r=.24, p<.05$; see Table 6$)$.

None of the participant demographics were significantly associated with patient selfefficacy for sex communication with physicians. However, data trends emerged $(p<.10)$ for ethnicity (minority participants having higher self-efficacy) and MS severity (participants with progressive MS and higher scores on the PDDS having higher self-efficacy). On the other hand, mental health variables were more consistently related to the PEPPI-S, including the total scale (an index of general distress) and all subscales but anxiety (which trended in that direction). Lower distress $(r=.30, p<.01)$, lower depressive symptoms $(r=.27, p<.05)$, more positive affect $(r=.27, p<.05)$, and more behavioral control $(r=.25, p<.05)$ were associated with high reported self-efficacy for sexual health communication with physicians. With regard to the overall sexual satisfaction, individuals with higher scores on the SSS (indicating greater sexual dissatisfaction) rated themselves as having lower self-confidence in communication about sex with physicians $(r=-37$, $p<.01)$.

We utilized hierarchical regression analyses to assess the contributions of PSQ patient satisfaction, time spent with the physician, and communication subscales on the frequency of sexual communication with a physician in the last year. Independent variables were chosen based on their significant relationships to our outcome. Demographic variables (age and relationship duration) were entered into our first block to account for variance explained by these factors, with our variables of interest entered in the second block. After accounting for demographic variables, our variables of interest accounted for an additional

Table 6 Correlates of frequency of sexual communication with physician in the past year

\begin{tabular}{|c|c|c|c|c|c|c|}
\hline Scale & 1 & 2 & 3 & 4 & 5 & 6 \\
\hline 1. Age & - & & & & & \\
\hline 2. Relationship duration & $.44 * *$ & - & & & & \\
\hline 3. Satisfaction with care (PSQ-18) & .06 & .11 & - & & & \\
\hline 4. Time spent with doctor (PSQ-18) & .06 & .17 & $.80 * *$ & - & & \\
\hline 5. Satisfaction w/communication (PSQ-18) & .07 & .17 & $.79 * *$ & $.84 * *$ & - & \\
\hline 6. Sex communication with physician, 12 months & $-.27 *$ & $-.29 *$ & $.37 * *$ & $.32 * *$ & $.24 *$ & - \\
\hline
\end{tabular}

$* p<.05 ; * * p<.01$ 
$14 \%$ of the variance in physician communication frequency [adj. $R^{2}=.18, F(5$, 53 ) $=3.60, p<.01]$, with patient satisfaction the sole significant contributor (see Table 7).

A second hierarchical regression analysis was conducted to assess the contribution of sexual satisfaction to the variance in patient's perceived efficacy for interacting with physicians around sexual health concerns, while controlling for the variance accounted for by mental health; this variable, as noted above, was significantly correlated with our efficacy variable. After controlling for mental health, sexual satisfaction accounted for an additional $10 \%$ of the variance in perceived efficacy $\left[\operatorname{adj} . R^{2}=.11, F(2,56)=4.66\right.$, $p<.05]$. Sexual satisfaction was a significant predictor of our outcome variable in this model $(\beta=-.36, t=-2.52, p<.05$; see Table 8$)$.

\section{Sexual Satisfaction}

None of our demographic variables were found to be associated with sexual satisfaction. However, data trends $(p<.10)$ emerged within several categories. Men trended toward higher sexual dissatisfaction than women $[t(59)=1.78, p=.08]$, as did those who were unemployed verses those who were employed $[t(57)=1.84, p=.07]$. In addition to the relationship between SSS and PEPPI-S (discussed in the previous section), sexual satisfaction was also associated with several sexual functioning, mental health, and patient satisfaction variables. Higher sexual dissatisfaction was reported by those reporting more sexual functioning problems as measured by the MSISQ-19 total $(r=.28, p<.05)$ as well as for the primary $(r=.30, p<.05)$ and tertiary $(r=.42, p<.001)$ subscales. Higher sexual dissatisfaction was associated with poor self-reported mental health on the general

Table 7 Predictors of frequency of sexual communication with providers

\begin{tabular}{lllll}
\hline Variable & $\beta$ & $t$ & Adjusted $R^{2}$ & $\Delta R^{2}$ \\
\hline Step 1 & & & .08 & $.11^{*}$ \\
$\quad$ Age & -.19 & -1.37 & & \\
$\quad$ Duration of relationship & -.21 & -1.53 & & $.14^{*}$ \\
Step 2 & & & .18 & \\
PSQ satisfaction & -.44 & $-2.10^{*}$ & & \\
PSQ time & -.12 & -.49 & & \\
PSQ communication & .22 & .89 & & \\
\hline
\end{tabular}

Table 8 Predictors of self-efficacy for communication about sex with providers

\begin{tabular}{llccr}
\hline Variable & $\beta$ & $t$ & Adjusted $R^{2}$ & $\Delta R^{2}$ \\
\hline Step 1 & & 1.65 & .03 & .05 \\
MHI & .21 & & .11 & $.10^{*}$ \\
Step 2 & & & \\
SSS & -.36 & $-2.52^{*}$ & & \\
\hline
\end{tabular}

Dependent variable: PEPPI-S

$* p<.05$ 
distress index $(r=-.51, p<.001)$ as well as all the MHI subscales. Higher sexual dissatisfaction was also correlated to lower patient satisfaction in the PSQ subscale $(r=-.30, p<.05)$.

A hierarchical regression analysis was conducted to assess the relative contributions of patient general satisfaction (as measured by the PSQ-18 General Satisfaction subscale) and patients' perceived efficacy for interacting with physicians around sexual health concerns on sexual satisfaction, accounting for both sexual dysfunction and mental health concerns. Sexual dysfunction was entered into our first block and accounted for $6 \%$ of the variance. Mental health concerns, entered into the second block, accounted for an addition $19 \%$ of the variance. When we entered our variables of interest, our overall model explained $30 \%$ of the variance in sexual satisfaction [adj. $\mathrm{R}^{2}=.29, F(4,54)=7.30, p<.01$ ]. Of our variables of interest, perceived efficacy for communicating with physicians about sexual concerns was the sole significant contributor to our model $(\beta=-.26, t=-2.24, p<.05$; see Table 9).

\section{Discussion}

The results of this study indicate that patients with MS report having sexual problems and believe they can talk with their physicians about them. Despite this, when asked about frequency, most patients do not talk to their physicians about these concerns. Of note, frequency of communication about sexual concerns was associated with physician variables related to satisfaction with care that were not specifically sexual health related, whereas self-efficacy for these interactions was associated with emotional health variables.

Most patients described some level of physical disability. Patients with progressive MS endorsed higher self-ratings of disability than individuals with other types of MS, which is consistent with literature stating individuals with progressive MS typically have a faster and more severe disease course [2]. Similarly, patients with progressive MS reported more sexual dysfunction than patients with other types of MS. Patients who had been diagnosed longer with MS reported higher self-ratings of disability, which is congruent with the literature reviewing disease course [5].

Patients with MS reported engaging in sexual activity at a frequency similar to adults in the United States [48], though the specifics of what these patients are doing with regard to sexual behaviors (touching vs. oral stimulation vs. penetrative sex) is unclear. However, it does not appear that patients' sexual dysfunction affects their attempts at sexual activity. Frequency of sexual functioning was not associated with MS severity, indicating that

Table 9 Predictors of sexual satisfaction

\begin{tabular}{|c|c|c|c|c|c|}
\hline $\begin{array}{l}\text { Table } 9 \text { Predictors of sexual } \\
\text { satisfaction }\end{array}$ & Variable & $\beta$ & $t$ & Adjusted $R^{2}$ & $\Delta R^{2}$ \\
\hline & Step 1 & & & .05 & $.07 *$ \\
\hline & MSISQ-19 & .26 & $2.06^{*}$ & & \\
\hline & Step 2 & & & .23 & $.19^{* *}$ \\
\hline & MHI & -.56 & $-3.81 * *$ & & \\
\hline & Step 3 & & & .30 & $.09 *$ \\
\hline \multirow{2}{*}{$\begin{array}{l}\text { Dependent variable: SSS } \\
* p<.05 ; * * p<.01\end{array}$} & PEPPI-S & -.26 & $-2.24 *$ & & \\
\hline & PSQ satisfaction & -.14 & -1.29 & & \\
\hline
\end{tabular}

Dependent variable: SSS

$* p<.05 ; * * p<.01$ 
sexual functioning is likely an important part of most patients' quality of life regardless of their disease progression.

Only one-third of patients reported talking to their physician in the past year about their sexual functioning, despite reports of sexual dysfunction. Discrepancies between beliefs and behaviors is a common finding in the literature [49]. Patients with a shorter relationship duration reported more communication about sexual functioning. Those patients may feel like it is important to negotiate many aspects of the relationship at the forefront rather than individuals in long-term relationships who may have already adapted to sexual difficulties. Moreover, patients at a younger age reported more frequent communication about sexual functioning in the past year, possibly because younger individuals are less likely to be in a long-term relationship or remain sexually exclusive [48]. Patients who reported having more general satisfaction with their medical care, more time spent with their physician, and higher satisfaction in their communication with their physician reported more frequently communicating with their physician about sexual health concerns. This indicates patients who feel their physician spends more time with them and are satisfied with their medical care may feel more comfortable introducing concerns than patients who feel their physician spends less time with them or are less satisfied with their medical care. The patient satisfaction variables in addition to the demographic variables accounted for twice as much variance in predicting frequency of sexual health related communication than the demographic variables alone; this result highlights the importance of patient satisfaction to communication about sexual health concerns.

Significant results were not found in regards to other demographic variables and patient self-efficacy for sexual communication with physicians or patient satisfaction, which may be influenced by on overall small sample size and the homogeneity of some aspects of the sample (e.g. all participants reside in the Southeastern region of the United States, $60.3 \%$ of sample Caucasian). However, data trends may be significant in a more heterogeneous sample. For example, minority patients tended to have a higher self-efficacy for sex communication. Perhaps there is cultural component that allows individuals to be more or less open about sexual communication. Additionally, there was a trend toward an association between patients with progressive MS and patients with a higher disability score as being more confident in their communication. Higher levels of dysfunction may provide greater opportunity to discuss symptoms with their physician or increased assertiveness; this hypothesis warrants further assessment. Conversely, patients with greater sexual dissatisfaction reported having lower self confidence in communication about sexual concerns with physicians. Patients may have greater sexual dissatisfaction because they are not comfortable addressing their concerns with physicians and thus do not receive appropriate treatment. Moreover, men had higher sexual dissatisfaction than women in this study, which is consistent with literature indicating sexual problems are related to physical health to a greater extent among men than women [49]. In addition, perhaps men have different expectations about sexual functioning than women or place it at a higher priority [50-52] as men in the general population report more frequent intercourse than women $[48,53,54]$. Unemployed patients reported higher levels of sexual dissatisfaction; this may be due to increased disability preventing them to engage in work and other activities (i.e., employed individuals may have overall higher functioning) or unemployment may result in decreased emotional functioning [55].

Individuals with greater mental health (e.g., less distress, lower depressive symptoms, more positive affect, and more behavioral control) reported higher self-efficacy in communication with physicians. This finding is consistent with research suggesting individuals who are psychologically healthier are likely more confident and have higher self-esteem 
[56]. Higher sexual dissatisfaction was associated with poorer mental health and lower patient satisfaction; this is consistent with findings relating mental health concerns such as depression with sexual problems [50]. Though causation cannot be determined in this study, both variables may have reciprocal effects (e.g., sexual dissatisfaction leads to increased depressive symptoms and increased depressive symptoms leads to sexual dissatisfaction). Additionally, patient's mental health and the patient satisfaction variables accounted for a significant amount of variance of the patient's sexual satisfaction; this result highlights the importance of patient's emotional functioning and their relationship with their physician on their self-reported sexual quality of life.

There are multiple limitations that bear consideration. For example, our patient population is relatively homogenous with regard to gender and geographic location. However, demographic variables were selectively heterogeneous related to self-rated level of disability and nearly $40 \%$ of the sample reported a minority racial category. Additionally, we are cautious to reach final conclusions due to the self-report nature of the study, which are subjective and likely to be affected by social desirability biases (e.g., patients reporting they are satisfied with the physician-patient relationship); employing the multitrait-multimethod matrix method [57] to reduce limitations of a single method of administration would be beneficial.

The results of this study have interesting clinical implications. By further understanding what influences a patient's ability to communicate about MS and its impact on sexuality, programs can be developed to facilitate effective communication among patients, their partners, and health care providers. Moreover, better communication may decrease MSrelated sexual functioning and intimacy concerns. Furthermore, results indicate that many factors are related to confidence in and frequency of communication; therefore, several different interventions may be beneficial.

Future research should investigate patients' priorities during a medical visit as a potential reason why they do not initiate a conversation about sexual health. For example, patients may prioritize pain over sexual functioning. Moreover, research should examine the frequency that physicians initiate a conversation about sexual concerns as well as reasons why they do not initiate this conversation (e.g., feel they are not trained about that area, do not prioritize sexual health, demographic factors, degrees of comfort in general, comfort related to specific topics). Subsequently, research should explore removing barriers to address patients' sexual concerns with physicians.

\section{Compliance with Ethical Standards}

Conflict of interest The authors declare that they have no conflict of interest.

Ethical Approval All procedures performed in studies involving human participants were in accordance with the ethical standards of the institutional and/or national research committee and with the 1964 Helsinki declaration and its later amendments or comparable ethical standards.

Informed Consent Informed consent was obtained from all individual participants included in the study.

\section{References}

1. Anderson, D.W., Ellenberg, J.H., Leventhal, C.M., Reingold, S.C., Rodriguez, M., Silberberg, D.H.: Revised estimate of the prevalence of multiple sclerosis in the United States. Ann. Neurol. 31, 333-336 (2004)

2. Aminoff, M.J., Greenberg, D.A., Simon, R.P.: Clinical Neurology, 6th edn. McGraw-Hill Medical, New York City (2005) 
3. Crayton, H., Heyman, R.A., Rossman, H.S.: A multimodal approach to managing the symptoms of multiple sclerosis. Neurology 63, S12-S18 (2004)

4. Philips, L.H., Henry, J.D., Nouzova, E., Cooper, C., Radlak, B., Summers, F.: Difficulties with emotion regulation in multiple sclerosis: links to executive function, mood, and quality of life. J. Clin. Exp. Neuropsychol. 36, 831-842 (2014)

5. Larocca, N.G.: Impact of walking impairment in multiple sclerosis: perspectives of patients and care partners. Patient 4, 189-201 (2011)

6. Kessler, T.M., Fowler, C.J., Panicker, J.N.: Sexual dysfunction in multiple sclerosis. Expert Rev. Neurother. 9(3), 341-350 (2009)

7. Wright, D.E., Stepleman, L.S., Davis, K.N., Hughes, M.D.: Development of a group educational intervention for sexual functioning and intimacy concerns in patients with MS. Int. J. MS Care 11, 107-113 (2009)

8. Zorzon, M., Zivadinov, R., Bosco, A., Monti Bragadin, L., Moretti, R., Bonfigli, L., Morassi, P., Iona, L.G., Cazzato, G.: Sexual dysfunction in multiple sclerosis: a case-control study. 1. Frequency and comparison of groups. Mult. Scler. 5(6), 418-427 (1999)

9. Demirkiran, M., Sarica, Y., Uguz, S., Yerdelen, D., Aslan, K.: Multiple sclerosis patients with and without sexual dysfunction: are there any differences? Mult. Scler. 12(2), 209-211 (2006)

10. Foley, F.: Introduction to intimacy and sexuality in MS. In: Uccelli, M.M. (ed.) MS in Focus: Intimacy and Sexuality, pp. 4-5. Cambridge Publishers, Cambridge (2005)

11. Jonsson, A.: Disseminated sclerosis and sexuality. Ugesker. Laeg. 165, 2642-2646 (2003)

12. Celik, D.B., Poyraz, E.Ç., Bingöl, A., İdiman, E., Özakbaş, S., Kaya, D.: Sexual dysfunction in multiple sclerosis: gender differences. J. Neurol. Sci. 324(1), 17-20 (2013)

13. Lew-Starowicz, M., Rola, R.: Prevalence of sexual dysfunctions among women with multiple sclerosis. Sex. Disabil. 31(2), 141-153 (2013)

14. Nortvedt, M.W., Riise, T., Frugard, J., et al.: Prevalence of bladder, bowel, and sexual problems among multiple sclerosis patients two to five years after diagnosis. Mult. Scler. 13, 106-112 (2007)

15. Sanders, A.S., Foley, F.W., LaRocca, N.G., Zemon, V.: The Multiple Sclerosis Intimacy and Sexuality Questionnaire-19 (MSISQ-19). Sex. Disabil. 18(1), 3-25 (2000)

16. Qaderi, K., Khoei, E.M.: Sexual problems and quality of life in women with multiple sclerosis. Sex. Disabil. 32(1), 35-43 (2014)

17. Schmidt, E.Z., Hofmann, P., Niederwieser, G., Kapfhammer, H.P., Bonelli, R.M.: Sexuality in multiple sclerosis. J. Neural Transm. 112, 1201-1211 (2005)

18. Foley, F.W., Iverson, J.: Sexuality and MS. In: Kalb, R.C., Scheinberg, L.C. (eds.) MS \& the Family. Demos Publications, New York (1992)

19. Foley, F., Sanders, A.: Sexuality, multiple sclerosis, and women. MS Manag. 4, 1-10 (1997)

20. Foley, F.W., LaRocca, N.G., Sanders, A.S., Zemon, V.: Rehabilitation of intimacy and sexual dysfunction in couples with multiple sclerosis. Mult. Scler. 7, 417-421 (2001)

21. Rubin, R.: Communication about sexual problems in male patients with multiple sclerosis. Nurs. Stand. 19(24), 33-37 (2005)

22. Flickinger, T.E., Saha, S., Roter, D., Korthuis, P. T., Sharp, V., Cohn, J., et al.: Respecting patients is associated with more patient-centered communication behaviors in clinical encounters. Patient Educ. Couns. 99, 220-226 (2016)

23. Loeb, D.F., Lee, R.S., Binswanger, I.A., Ellison, M.C., Aagaard, E.M.: Patient, resident physician, and visit factors associated with documentation of sexual history in the outpatient setting. J. Gen. Intern. Med. 26(8), 887-893 (2011)

24. Meystre-Agustoni, G., Jeannin, A., de Heller, K., Pécoud, A., Bodenmann, P., Dubois-Arber, F.: Talking about sexuality with the physician: are patients receiving what they wish. Swiss Med. Wkly. 141, w13178 (2011)

25. Gilbert, E., Perz, J., Ussher, J.M.: Talking about sex with health professionals: the experience of people with cancer and their partners. Eur. J. Cancer Care 25, 280-293 (2016)

26. Burke, L.M.: Sexual dysfunction following radiotherapy for cervical cancer. Br. J. Nurs. 5, 239-244 (1996)

27. Gianotten, W.L., Bender, J.L., Post, M.W., Mechtild, H.: Training in sexology for medical and paramedical professionals: a model for the rehabilitation setting. Sex. Relation Ther. J. 21, 303-317 (2006)

28. Butler, L., Banfield, V., Sveinson, T., Allen, K.: Conceptualizing sexual health in cancer care. West. J. Nurs. Res. 20, 683-705 (1998)

29. Barni, S., Mondin, R.: Sexual dysfunction in treated breast cancer patients. Ann. Oncol. 8, 149-153 (1997) 
30. Hordern, A.J., Street, A.F.: Communicating about patient sexuality and intimacy after cancer: mismatched expectations and unmet needs. Med. J. Aust. 186, 224-227 (2007)

31. Kroenke, K., Jackson, J.L., Chamberlin, J.: Depressive and anxiety disorders in patients presenting with physical complaints: clinical predictors and outcome. Am. J. Med. 103, 339-347 (1997)

32. Henschke, N., Wouda, L., Maher, C.G., Hush, J.M., van Tulder, M.W.: Determinants of patient satisfaction 1 year after presenting to primary care with acute low back pain. Clin. J. Pain 29(6), 512-517 (2013)

33. Jackson, J.L., Kroenke, K.: Difficult patient encounters in the ambulatory clinic: clinical predictors and outcomes. Arch. Intern. Med. 159, 1069-1075 (1999)

34. Groom, M.J., Lincoln, N.B., Francis, V.M., Stephan, T.F.: Assessing mood in patients with multiple sclerosis. Clin. Rehabil. 17, 847-857 (2003)

35. Lester, K., Stepleman, L.M., Hughes, M.: The association of illness severity, self-reported cognitive impairment, and perceived illness management with depression and anxiety in a multiple sclerosis clinic population. J. Behav. Med. 30, 177-186 (2007)

36. Schiffer, R.B., Caine, E.D., Bamford, K.A., Levy, S.: Depressive episodes in patients with multiple sclerosis. Am. J. Psychiatry 140, 1498-1500 (1983)

37. Aikens, J.E., Fischer, J.S., Namey, M., Rudick, R.A.: A replicated prospective investigation of life stress, coping, and depressive symptoms in multiple sclerosis. J. Behav. Med. 20, 433-445 (1997)

38. Joffe, R.T., Lippert, G.P., Gray, T.A., Sawa, G., Horvath, Z.: Mood disorder and multiple sclerosis. Arch. Neurol. 44, 376-378 (1987)

39. Minden, S.L., Orav, J., Reich, P.: Depression in multiple sclerosis. Gen. Hosp. Psychiatry 9, 426-434 (1987)

40. Hohol, M., Orav, E., Weiner, H.: Disease steps in multiple sclerosis: a simple approach to evaluate disease progression. Neurology 45(2), 251-255 (1995)

41. Suh, Y., Moti, R.W., Mohr, D.C.: Physical activity, disability, and mood in the early stage of multiple sclerosis. Disabil. Health J. 3(2), 93-98 (2010)

42. Veit, C.T., Ware, J.E.: The structure of psychological distress and well-being in general populations. J. Consult. Clin. Psychol. 51(5), 730-742 (1983)

43. Ritvo, P.G., Fischer, J.S., Miller, D.M., Andrews, H., Paty, D.W., LaRocca, N.G.: Multiple Sclerosis Quality of Life Inventory: A User's Manual. National Multiple Sclerosis Society, New York (1997)

44. Sanders, A., Foley, F.W., LaRocca, N.G., Zemon, V.: The Multiple Sclerosis Intimacy and Sexuality Questionnaire-19. Sex. Disabil. 18(1), 3-26 (2000)

45. Nowinski, J.K., LoPiccolo, J.: Assessing sexual behaviors in couples. J. Sex Marital Ther. 5, 225-243 (1979)

46. Marshall, G.N., Hays, R.D.: The Patient Satisfaction Questionnaire Short-Form (PSQ-18). Rand Corp, Santa Monica (1994)

47. Maly, R.C., Frank, J.C., Marshall, G.N., DiMatteo, M.R., Reuben, D.B.: Perceived efficacy in patientphysician interactions in older persons. J. Am. Geriatr. Soc. 46(7), 889-894 (1998)

48. Seidman, S.N., Rieder, R.O.: A review of sexual behavior in the United States. Am. J. Psych. 151, 330-341 (1994)

49. Ajzen, I., Timko, C.: Correspondence between health attitudes and behavior. Basic Appl. Soc. Psychol. 7, 259-276 (1986)

50. Laumann, E.O., Nicolosi, A., Glasser, D.B., Paik, A., Gingell, C., Moreira, E., Wang, T.: Sexual problems among women and men aged 40-80 y: prevalence and correlates identified in the global study of sexual attitudes and behaviors. Int. J. Impot. Res. 17, 39-57 (2005)

51. Geer, J.H., Robertson, G.G.: Implicit attitudes in sexuality: gender differences. Arch. Sex. Behav. 34, 671-677 (2005)

52. Oliver, M.B., Hyde, J.S.: Gender differences in sexuality: a meta-analysis. Psychol. Bull. 114, 29-51 (1993)

53. Petersen, J.L., Hyde, J.S.: A meta-analytic review of research on gender differences in sexuality, 1993-2007. Psychol. Bull. 136, 21-38 (2010)

54. Hopcroft, R.L.: Sex, status, and reproductive success in the contemporary United States. Evol. Hum. Behav. 27, 104-120 (2006)

55. Linn, M.W., Sandifer, R., Stein, S.: Effects of unemployment on mental and physical health. Am. J. Public Health 75, 502-506 (1985)

56. Cheng, H., Furnham, A.: Personality, self-esteem, and demographic predictions of happiness and depression. Pers. Individ. Dif. 34, 921-942 (2003)

57. Campbell, D.T., Fiske, D.W.: Convergent and discriminant validation by the multitrait-multimethod matrix. Psychol. Bull. 56, 81-105 (1959) 